million) US children aged 5 to 18 received MPH for ADD in 1995 . The increased usage was related to more prolonged treatment, more girls, and adolescents receiving medication for ADD. (Safer DJ, Zito JM, Fine EM. Increased methylphenidate usage for attention deficit disorder in the 1990s. Pediatrics Dec 1996;98:1084-1088). (Reprints: Daniel J Safer MD, 7702 Dunmanway, Dundalk, MD 21222).

COMMENT. A 2.8-fold increase in methylphenidate usage between 1990 and 1995, as shown in this study, is far less than the media claims of a 6-fold increased usage, based on DEA production quotas for methylphenidate in this time period. The findings do not address the appropriateness of stimulant therapy for ADHD.

\title{
SLEEP DISORDERS IN NEUROLOGICAL PRACTICE
}

Pediatric sleep disorders are reviewed from the Infant and Family Development Laboratory, Department of Psychiatry and Graduate Program in Human Development, University of California, Davis. Sleep disorders associated with neurological disorders include: 1) sleep-related epilepsy; 2) sleep-related headaches; 3) degenerative disorders; 4) developmental disorders (Down syndrome; Prader-Willi syndrome); 5) methylphenidate treatment for ADHD causing sleep disturbance; and 6) Kleine-Levin syndrome, with episodic excessive somnolence. Medical and psychiatric disorders that interfere with sleep include: asthma, gastroesophageal reflux, psychoses, mood disorders, anxiety, and substance abuse disorders. Primary sleep disorders include dyssomnias (obstructive sleep apnea with enlarged tonsils and adenoids, narcolepsy, extrinsic sleep irregularities of infants, circadian rhythm sleep disorders); parasomnias (sleep terrors, sleepwalking, head banging, rocking, talking, leg cramps, nightmares, bruxism, enuresis). (Anders TF, Eiben LA. Pediatric sleep disorders; a review of the past 10 years. I Am Acad Child Adolesc Psychiatry Jan 1997;36:9-20). (Reprints: Dr Anders, Department of Psychiatry, UCDMC, 4430 V Street, Sacramento, CA 95817).

COMMENT. The pediatric neurologist may be consulted because of REM parasomnias and the differentiation of nightmares, sleep terrors, and nocturnal frontal lobe seizures. EEGs are frequently nonspecific, and videopolysomnographic monitoring is often necessary. A trial of anticonvulsant medication, eg clonazepam, may be warranted in suspected but unconfirmed cases of seizures. (see Ped Neur Briefs Oct 1996 for reports of nocturnal frontal lobe epilepsy and differential diagnosis).

\section{LANGUAGE DISORDERS}

\section{ACQUIRED NEUROLOGIC MUTISM}

The behavioral features of four children with acquired neurologic mutism are reported from the Department of Neurology, University Hospital Rotterdam-Dijkzigt, Rotterdam; and Department of Medical Psychology, Ziekenhuis Walcheren, Vlissingen, The Netherlands. Neuropsychologic examinations revealed changes in phonation, orofacial movements, communicative behavior, and linguistic functions. Case 1, akinetic mutism, post severe head injury, hemorrhage in left fronto-parietal area, spastic quadriplegia, motionless except for slow eye movements and head turning when addressed, no orofacial movements except smacking of lips, recovered 
slow dysarthric speech after 15 months; word finding unimpaired; Case 2, aphasic mutism, post head injury coma and contusion of left hemisphere, right flaccid hemiparesis, mobility improved in 6 weeks, but aphasia persisted; Case 3 , cortical deafness, post head trauma neglect for verbal and nonverbal auditory stimuli, recovery of awareness of language and sounds after 5 weeks, but unaware of meanings (auditory agnosia), responded to written words, complete recovery with normal language comprehension after 11 weeks; Case 4, posterior fossa mutism, post craniotomy and removal of medulloblastoma, recovery of speech after 5 weeks. (van Mourik M, van Dongen HR, CatsmanBerrevoets CE. The many faces of acquired neurologic mutism in childhood. Pediatr Neurol Nov 1996;15:352-357). (Respond: Dr van Mourik, Department of Medical Psychology, Ziekenhuis Walcheren, Postbus 3200, 4380 DD Vlissingen, The Netherlands).

COMMENT. The various etiologies of acquired mutism may be differentiated by a battery of neuropsychological tests for phonation, orofacial movements, communication, and linguistic functions. In addition to the neurologic examination and neuroimaging, an EEG should distinguish Landau-Kleffner syndrome, a further example of mutism in some cases.

\section{COMMUNICATION DEVELOPMENT IN RETT SYNDROME}

The development of linguistic communication in 17 children with Rett syndrome was investigated by a parent questionnaire based on the Clinical Linguistic Auditory Milestone Scale in a study at the Department of Speech Pathology and Audiology, University of Alberta, Edmonton, Canada. From birth to 24 months, no child exceeded the stage of single word utterances, with a maximum of 4-6 single words, such as mama/dada. This milestone represents the transition from babbling to real words, normally occurring before the end of the first year. Gesture milestones involving pointing or showing and the beginning of communication were largely absent. Onset of regression had occurred for 14 of the 17 children. Sixteen children exhibited hand movement symptoms, generally during the second year of life. (Tams-Little S, Holdgrafer G. Early communication development in children with Rett syndrome. Brain Dev Sept/Oct 1996;18:376-378). (Respond: Fax: (1) (403) 492-1626).

COMMENT. These findings contradict the observation that communication development in Rett syndrome before one year is essentially normal. Children with Rett syndrome may develop language to the mama/dada and other single word stage, but limited intentional gestural communication and lack of finger pointing is a potential early predictor of Rett syndrome.

Pervasive developmental disorders as a further cause of deficits in language and communication skills are reviewed from the Department of Neurology, Miami Children's Hospital, FL. (Tuchman R. Pervasive developmental disorder. Neurological perspective. Acta Neuropediatr 1996;2:8293). Of 421 children with PDD, referred because of language and behavioral disorders, $11 \%$ had chromosomal disorders, genetic syndromes, and familial occurrence. Motor deficits occurred in $18 \%$, sleep problems in $20 \%$, stereotypies in $71 \%$, and regression of language in $28 \%$. Abnormal EEGs were found in $29 \%$, epilepsy in $13 \%$, and MRI abnormalities in $19 \%$. No patients in this study had classical Rett syndrome; $5 \%$ of girls had a "forme fruste" of Rett syndrome. Since the diagnosis of Rett syndrome is based on clinical criteria, girls presenting with autism should always be considered for possible Rett syndrome. 